\title{
Thyroid incidentalomas in patients with multiple endocrine neoplasia type 1
}

\author{
Lutske Lodewijk ${ }^{*}$, Pim J Bongers ${ }^{*}$, Jakob W Kist, Elfi B Conemans, Joanne M de Laat, \\ Carla R C Pieterman, Anouk N A van der Horst-Schrivers ${ }^{1}$, Ciska Jorna ${ }^{1}$, \\ Ad R Hermus ${ }^{2}$, Olaf M Dekkers ${ }^{3}$, Wouter W de Herder ${ }^{4}$, Madeleine L Drent ${ }^{5}$, \\ Peter H Bisschop ${ }^{6}$, Bas Havekes', Inne H M Borel Rinkes, Menno R Vriens \\ and Gerlof D Valk ${ }^{\dagger}$
}

\begin{abstract}
Department of Oncology, University Medical Center Utrecht, 3508 GA Utrecht, The Netherlands, ${ }^{1}$ Departments of Endocrinology and Surgery, University Medical Center Groningen, 9700 RB Groningen, The Netherlands,

${ }^{2}$ Department of Endocrinology, Radboud University Medical Center, 6500 HB Nijmegen, The Netherlands,

${ }^{3}$ Departments of Endocrinology and Metabolism and Clinical Epidemiology, Leiden University Medical Center, 2300 RC Leiden, The Netherlands, " Department of Internal Medicine, Erasmus Medical Center, 3000 CA Rotterdam, The Netherlands, ${ }^{5}$ Department of Internal Medicine, VU University Medical Center, 1007 MB Amsterdam, The Netherlands, ${ }^{6}$ Department of Endocrinology and Metabolism, Academic Medical Center, 1100 DD Amsterdam, The Netherlands and ${ }^{7}$ Department of Internal Medicine, Division of Endocrinology, Maastricht University Medical Center, 6202 AZ Maastricht, The Netherlands

${ }^{\dagger} \mathrm{G}$ D Valk is now at Divison of Oncology, University Medical Center Utrecht, Heidelberglaan 100, 3584 CX Utrecht, The Netherlands

*(L Lodewijk and P J Bongers contributed equally to this work)
\end{abstract}

Correspondence should be addressed to G D Valk

Email

G.D.Valk@umcutrecht.nl

\begin{abstract}
Objective: Currently, little is known about the prevalence of thyroid tumors in multiple endocrine neoplasia type 1 (MEN1) patients and it is unclear whether tumorigenesis of these thyroid tumors is MEN1-related. The aim of the study was to assess the prevalence of thyroid incidentalomas in MEN1 patients compared with nonMEN1 patients and to verify whether thyroid tumorigenesis is MEN1-related.
\end{abstract}

Design: A cross-sectional study.

Methods: The study included two groups: patients with MEN1 and a matched non-MEN1 control group without known thyroid disease, who underwent an ultrasound of the neck for the localization of parathyroid adenoma. Ninety-five MEN1 patients underwent ultrasound of the neck and were matched on gender and age with non-MEN1 patients. The prevalence of thyroid incidentalomas described in the ultrasound report was scored. Multinodular goiters, solitary nodes, and cysts were scored as incidentalomas. Presence of nuclear menin expression was evaluated by menin immunostaining of the thyroid tumors.

Results: In the MEN1 group, 43 (45\%) patients had a thyroid incidentaloma compared with 48 (51\%) in the non-MEN1 group, of which $14(15 \%)$ and $16(17 \%)$, respectively, were solitary nodes. Menin was expressed in the nuclei of all evaluated thyroid tumors.

Conclusions: MEN1 patients do not have a higher prevalence of thyroid incidentalomas compared with primary hyperparathyroidism patients without the diagnosis of MEN1. Menin was expressed in the thyroid tumors of MEN1 patients.

\section{Introduction}

Multiple endocrine neoplasia type 1 (MEN1) syndrome is characterized by the combined occurrence of pituitary tumors, primary hyperparathyroidism (pHPT), pancreatic and duodenal neuroendocrine tumors (NET), adrenal adenomas, and NETs of stomach, lung, and thymus (1). Recently, MEN1 has also turned out to be a breast cancer (c) 2015 European Society of Endocrinology Printed in Great Britain
Published by Bioscientifica Ltd. 
susceptible syndrome (2). The syndrome is caused by an inactivating germline mutation in the MEN1 gene, which encodes for the tumor suppressor protein menin. Tumorigenesis of MEN1-related tumors is characterized by loss of menin expression or the production of nonfunctional menin in case of missense (or in-frame) alterations of the MEN1 gene (3). At present, little is known about the prevalence of thyroid tumors in MEN1 patients. Marx et al. found a prevalence of $12 \%$ thyroid tumors ( $8 \%$ follicular adenoma and 5\% papillary thyroid carcinoma) in 130 MEN1 patients. These patients were screened for all types of endocrine abnormalities (4). The recently published MEN1 guideline reports that thyroid tumors (adenomas, colloid goiters, and carcinomas) occur in more than $25 \%$ of patients with MEN1. Subsequently, the guideline states that 'because of the high prevalence of thyroid abnormalities in the general population, the association of thyroid abnormalities with MEN1 may be incidental and not significant' (1). However, the lack of evidence regarding the clinical relevance of thyroid tumors might cause an extra dilemma for both endocrinologist and endocrine surgeon treating patients with MEN1.

Primary hyperparathyroidism (pHPT) occurs in 90\% of the MEN1 patients. Therefore, a substantial part of this population undergoes a neck ultrasound to localize parathyroid adenomas (5). Because of the anatomical relationship between thyroid and parathyroid glands, it is inevitable that the thyroid is imaged during the neck ultrasound, which increases the chance of incidentally finding a thyroid tumor.

The aim of this study was to assess the prevalence of thyroid incidentalomas in the Dutch MEN1 population compared with a matched reference group of non-MEN1 patients. To support the epidemiologic findings, we studied menin expression in thyroid tumors of MEN1 patients by immunohistochemistry to assess whether loss of nuclear menin was present.

\section{Subjects and methods}

\section{Study group}

All MEN1 patients in the Dutch MEN1 Study Group (DMSG) database were identified as described previously (325 patients) (6). From this database, data regarding demographics, mutation status (according to the Human Genome Variation Society nomenclature) (7), MEN1 manifestations, imaging, surgery, and histology reports were extracted. For further analysis, patients were selected who had a neck ultrasound because of PHPT in which the thyroid was described (102 patients; Fig. 1). The baseline characteristics of 102 patients were compared with the other MEN1 patients to verify whether it was a representative subgroup (Table 1).

As a non-MEN1 reference group, 201 consecutive patients who underwent neck ultrasound between 2003 and 2012 for pHPT, not having MEN1 or known thyroid disease, were identified from the hospital radiology database of the University Medical Centers of Utrecht and Groningen in The Netherlands. This reference group will further be referred to as the non-MEN1 group. As age and gender differed significantly in the MEN1 and the non-MEN1 groups, patients were matched (1:1) on these variables via the 'case-control matching' extension in SPSS. For age, a spread of 3 years was accepted for the matching. In total, 95 patients could be matched. Seven MEN1 patients had to be excluded because no match was available. These consisted of five females and two men with a median age of 21 years, ranging from 15 to 33 years. Of those seven patients, two patients had a cyst.

Multinodular goiters, solitary nodes, and cysts that were identified by the ultrasounds of the neck were scored. By definition these tumors are incidentalomas.

\section{Immunohistochemistry}

As a proxy, for menin expression, immunohistochemistry was performed on formalin-fixed paraffin-embedded (FFPE) tissues from five thyroid samples and a negative control sample. However, certain types of the MEN1 mutations do not lead to an altered expression of menin, but due to these mutations there is a nonfunctional protein. Therefore, we listed the mutations per sample in Supplementary Table 1 , see section on supplementary data

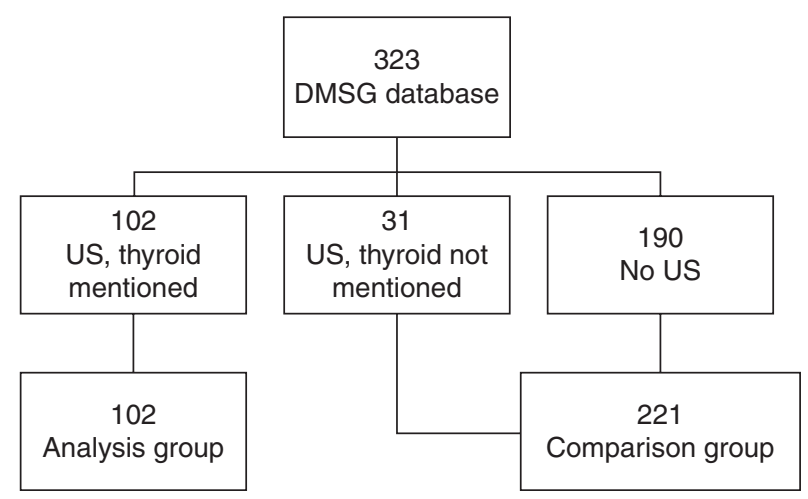

\section{Figure 1}

Flowchart of patients from the DMSG database. US, neck ultrasound. 
Table 1 Baseline comparison between MEN1 patients with or without neck ultrasound.

\begin{tabular}{l} 
\\
\hline Female, $n(\%)$ \\
Age, mean in years (s.D.) \\
Follow-up, mean in years (s.D.) \\
pHPT, $n(\%)$ \\
Type of mutation, $n(\%)$ \\
Clinical diagnoses ${ }^{\mathrm{a}}$ \\
Nonsense \\
Missense \\
Frameshift \\
Splice \\
Unclassified \\
Large deletions $^{\mathrm{b}}$ \\
Unknown $^{\mathrm{c}}$
\end{tabular}

\begin{tabular}{c}
\hline Comparison group $(n=221)$ \\
\hline $120(54.3)$ \\
$46.7(16.5)$ \\
$10.2(9.9)$ \\
$161(73.6)$ \\
$15(6.8)$ \\
$30(13.6)$ \\
$43(19.5)$ \\
$68(30.8)$ \\
$12(5.4)$ \\
$2(0.9)$ \\
$48(21.7)$ \\
$3(1.4)$
\end{tabular}

\begin{tabular}{ccc}
\hline Analysis group $(n=102)$ & & P value \\
\hline $68(66.7)$ & & 0.04 \\
$51.9(14.8)$ & & 0.01 \\
$10.5(8.5)$ & 0.82 \\
$102(100.0)$ & 0.00 \\
$15(14.7)$ & 0.29 \\
$18(17.6)$ & \\
$18(17.6)$ & \\
$32(31.4)$ & \\
$2(2.0)$ & \\
$0(0.0)$ & \\
$15(14.7)$ & \\
$2(2.0)$ & \\
\hline
\end{tabular}

pHPT, primary hyperparathyroidism.

${ }^{a}$ Clinical diagnoses are patients with two or more of the major manifestations of MEN1 without a germline mutation.

b Large deletions include in-frame deletions, deletions of exon 1 and 2, deletions of exon 1, 2, and 3, and deletions of the entire MEN1 gene.

'Unknown consists of patients with clinical diagnosis of MEN1 in whom either no genetic testing is performed or the exact location of the mutation is unknown.

given at the end of this article. All thyroid tumors were selected and evaluated by a dedicated pathologist (PJvD). As a negative control, we used a sample in which, by sequencing of the DNA, loss of heterozygosity (LOH) was proven. This sample was from a patient with infiltrative ductal carcinoma of the breast with a germline nonsense mutation (c.377G $>$ A(p.Trp126X)) (2). All tissues were sampled from surgical specimen according the standard procedure in the University Medical Center Utrecht. The slides were deparaffinized with xylene and rehydrated in decreasing ethanol dilutions. Endogenous peroxidase activity was blocked with 3\% hydrogen peroxide. Antigen retrieval was achieved by boiling slides in citrate buffer (pH 6.0) for $20 \mathrm{~min}$. The slides were then incubated with the rabbit polyclonal antibody against menin (Menin, A300-105A, Bethyl Laboratories, Inc., Montgomery, TX, USA), diluted at $1: 1600$ for $1 \mathrm{~h}$ at room temperature. For detection of primary antibodies, goat anti-mouse poly-HRP (Powervision, Immunologic, Immunovision Technologies, Brisbane, CA, USA) was used. All slides were developed with diaminobenzidine (DAB). The slides were counterstained with filtered hematoxylin, dehydrated through a graded series of ethanol, immersed in xylene, and mounted. Menin staining was reviewed by an experienced pathologist and compared with the negative control.

\section{Statistical analyses}

Continuous variables are expressed as means with a s.D. if normally distributed and as median (25 and $75 \%$ percentile) if not. Categorical and dichotomous variables are expressed as absolute numbers (\%). Matching was performed by the case-control matching function available in SPSS. Student's $t$-test, Mann-Whitney $U$ test, and Pearson's $\chi^{2}$ test were used where appropriate. Statistical significance was reached when $P$ value was smaller than 0.05 . Calculations were performed using SPSS/PC version 23.0.

\section{Results}

\section{Baseline comparison}

The presence of thyroid was mentioned in the report of the neck ultrasound in 102 patients (32\%) of a total of 323 MEN1 patients. In 31 (10\%) patients, an ultrasound was performed but the presence of thyroid was not mentioned in the report. No ultrasound was performed between 1990 and 2010 in 190 (59\%) MEN1 patients (Fig. 1). Patient characteristics of the groups with and without a neck ultrasound were compared with baseline characteristics

Table 2 Thyroid incidentalomas in MEN1 patients compared with a matched control group.

\begin{tabular}{|c|c|c|c|}
\hline & $\begin{array}{l}\text { MEN1 } \\
(n=95)\end{array}$ & $\begin{array}{c}\text { Non-MEN1 } \\
(n=95)\end{array}$ & $P$ value \\
\hline Female, $n(\%)$ & $63(66)$ & $63(66)$ & - \\
\hline $\begin{array}{l}\text { Age at date of ultrasound, } \\
\text { mean (s.D.) }\end{array}$ & $48.3(14.3)$ & $46.6(13.8)$ & - \\
\hline Incidentaloma, $n(\%)$ & $43(45)$ & $48(51)$ & NS \\
\hline Multinodular goitre, $n(\%)$ & $25(26)$ & $29(31)$ & NS \\
\hline Solitary node, $n(\%)$ & $14(15)$ & $16(17)$ & NS \\
\hline Cyst, $n(\%)$ & $4(4)$ & $4(4)$ & NS \\
\hline
\end{tabular}


Table 3 Diagnoses of the thyroid tumors after histologic examination in MEN1 patients.

\section{Histologic examinations} carcinoma

Micro-invasive follicular thyroid carcinoma Follicular adenoma

Multinodular goiter

Nodular dysplasia

Nodular hyperplasia

Lymphocytic thyroiditis
Multifocal micro-invasive medullary thyroid

\begin{tabular}{c}
\hline $\boldsymbol{n}=\mathbf{1 7}$ \\
\hline 1 \\
1 \\
4 \\
2 \\
5 \\
3 \\
1
\end{tabular}

(Table 1). The group that underwent ultrasound of the neck consisted of more female patients (68 (66.7\%) vs $120(54.3 \%))$ and was significantly older (51.9 (14.8) vs 46.7 (16.5)). There was no difference in mean follow-up time and the type of mutation between the groups with and without a neck ultrasound.

\section{Thyroid incidentalomas}

In 43 MEN1 patients (45\%) and 48 non-MEN1 patients (51\%), incidentalomas of the thyroid were found on neck ultrasound. The tumors consisted of 25 (26\%) and 29 (31\%) multinodular goiters, 14 (15\%) and 16 (17\%) solitary nodes, four (4\%) and four (4\%) cysts in the MEN1 group and the non-MEN1 group, respectively.
No significant differences were found (Table 2). When reported, size of the solitary nodes was also analyzed. The median size of the solitary nodes was $6 \mathrm{~mm}$ (Interquartile range (IQR) $4.5-11 \mathrm{~mm}$ ) in the MEN1 group and $8 \mathrm{~mm}$ (IQR 4.0-9.0 $\mathrm{mm}$ ) in the non-MEN1 group ( $P$ value 0.94$)$.

\section{Thyroid histology}

From 17 MEN1 patients, the histology reports of the thyroid tumors were available and the diagnoses are given in Table 3. Follicular adenomas and nodular dysplasia were most prevalent. Immunohistochemistry was performed in a representative subset of the different types of thyroid tumors. In all thyroid tumors, we tested whether menin was present by immunohistochemical staining of the nucleus of adjacent normal and tumor tissues. In the control sample, no menin expression was found, indicating loss of heterozygosity (Fig. 2 and Supplementary Figure 1, see section on supplementary data given at the end of this article).

\section{Discussion}

The results of this study show that the prevalence of thyroid incidentalomas in patients with MEN1 is equal to a matched reference group with non-MEN1 patients. These results are in-line with the suggestion in the

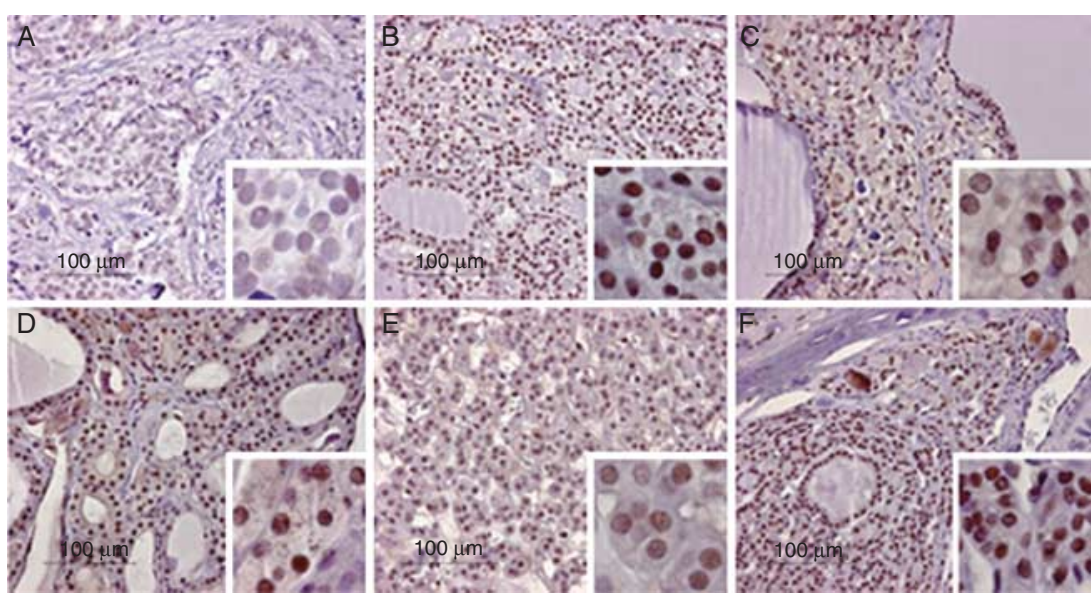

\section{Figure 2}

Immunohistochemical analysis of menin protein expression in the nuclei of five thyroid tumors from MEN1 patients and absence of expression in one infiltrating ductal carcinoma of the breast from a MEN1 patient with proven LOH. Pictures are taken with a $20 \times$ magnification, inlays with $40 \times$ magnification. (A) Infiltrative ductal carcinoma of the breast; (B) hyperplastic node; (C) microinvasive medullary thyroid carcinoma; (D) multinodular goiter; (E) follicular adenoma; and $(F)$ microinvasive follicular thyroid carcinoma. 
recently updated guideline, that the high percentage (25\%) of thyroid tumors occurring in MEN1 patients is incidental and not significant (1). These epidemiologic results are strongly supported by the immunohistochemistry, which show a positive menin staining indicating the presence of intact nuclear menin expression in a representative subset of thyroid tumors found in patients with MEN1.

The non-MEN1 patients were considered the best available control group facing the fact that a neck ultrasound was performed for the same indication as in the MEN1 patients. As MEN1 patients present with pHPT at a young age, not all MEN1 patients could be matched. Also in this young patient group, a very low prevalence of thyroid incidentalomas was found which is in-line with what one can expect in the general population.

In literature, prevalence rates for solitary nodes in healthy individuals are around 10\% compared with 15\% in our study $(8,9,10,11)$. Owing to the retrospective character of the study, all patients $(n=31)$ who underwent a neck ultrasound in which the thyroid was not mentioned in the report were excluded. If we assume that there was no solitary node in those 31 neck ultrasounds, our prevalence would be similar (11\%) to the prevalence rates reported in literature.

From 17 MEN1 patients, histology reports were available from thyroid tumors found by ultrasound. Of those 17 patients, one patient had a microinvasive medullary thyroid carcinoma and one patient had a microinvasive follicular thyroid carcinoma, and the other 15 showed benign pathology. MEN1-related tumors are characterized by loss of the second allele of MEN1 gene, encoding for the protein menin, resulting in no functional copies of the gene (12). In four case series of MEN1 patients with thyroid carcinoma, loss of heterozygosity ( $\mathrm{LOH})$ was examined. The results did not show any $\mathrm{LOH}$ which indicates no etiological relation between the presence of MEN1 mutation and thyroid carcinoma $(13,14,15,16)$. We assessed loss of menin expression by immunohistochemistry in a representative subset of diagnoses; in all evaluated tissue menin was expressed throughout the tumor and adjacent normal thyroid tissue. This indicates that there is no haploinsufficiency, i.e. the intact copy of the MEN1 gene produces enough protein to bring about a WT condition.

It is a clinical challenge for both endocrinologists and surgeons to deal with thyroid incidentalomas in MEN1 patients. On the one hand, the burden of the patient needs to be as low as possible, and on the other hand, malignancies need to be identified and treated as early as possible. Our results indicate that in case of a thyroid incidentaloma in MEN1 patients, prevailing guidelines for thyroid incidentalomas in the general population can be followed.

In conclusion, our results show no difference in the prevalence of thyroid incidentalomas in MEN1 patients compared with patients with pHPT without the diagnosis of MEN1. The epidemiologic findings were validated by menin expression in the nuclei.

\section{Supplementary data}

This is linked to the online version of the paper at http://dx.doi.org/10.1530/ EJE-14-0897.

\section{Declaration of interest}

The authors declare that there is no conflict of interest that could be perceived as prejudicing the impartiality of the research reported.

\section{Funding}

This research did not receive any specific grant from any funding agency in the public, commercial or not-for-profit sector.

\section{Acknowledgements}

The authors thank Paul J van Diest for scoring of the menin staining.

\section{References}

1 Thakker RV, Newey PJ, Walls GV, Bilezikian J, Dralle H, Ebeling PR, Melmed S, Sakurai A, Tonelli F, Brandi ML et al. Clinical practice guidelines for multiple endocrine neoplasia type 1 (MEN1). Journal of Clinical Endocrinology and Metabolism 201297 2990-3011. (doi:10.1210/ jc.2012-1230)

2 Dreijerink KM, Goudet P, Burgess JR, Valk GD \& International Breast Cancer in MENSG. Breast-cancer predisposition in multiple endocrine neoplasia type 1. New England Journal of Medicine 2014371 583-584. (doi:10.1056/NEJMc1406028)

3 Lemmens I, Van de Ven WJ, Kas K, Zhang CX, Giraud S, Wautot V, Buisson N, De Witte K, Salandre J, Lenoir G et al. Identification of the multiple endocrine neoplasia type 1 (MEN1) gene. The European Consortium on MEN1. Human Molecular Genetics 19976 1177-1183. (doi:10.1093/hmg/6.7.1177)

4 Marx S, Spiegel AM, Skarulis MC, Doppman JL, Collins FS \& Liotta LA. Multiple endocrine neoplasia type 1: clinical and genetic topics. Annals of Internal Medicine 1998129 484-494. (doi:10.7326/0003-4819. 129-6-199809150-00011)

5 Thakker RV. Multiple endocrine neoplasia type 1 (MEN1). Best Practice \& Research. Clinical Endocrinology \& Metabolism 201024 355-370. (doi:10.1016/j.beem.2010.07.003)

6 de Laat JM, Pieterman CR, Weijmans M, Hermus AR, Dekkers OM, de Herder WW, van der Horst-Schrivers AN, Drent ML, Bisschop PH, Havekes B et al. Low accuracy of tumor markers for diagnosing pancreatic neuroendocrine tumors in multiple endocrine neoplasia 
type 1 patients. Journal of Clinical Endocrinology and Metabolism 201398 4143-4151. (doi:10.1210/jc.2013-1800)

7 Cotton RG \& Horaitis O. The HUGO Mutation Database Initiative, Human Genome Organization. Human Genome Organization. Pharmacogenomics Journal 20022 16-19. (doi:10.1038/sj.tpj.6500070)

8 Jin J, Wilhelm SM \& McHenry CR. Incidental thyroid nodule: patterns of diagnosis and rate of malignancy. American Journal of Surgery 2009 197 320-324. (doi:10.1016/j.amjsurg.2008.10.006)

9 Reiners C, Wegscheider K, Schicha H, Theissen P, Vaupel R, Wrbitzky R \& Schumm-Draeger PM. Prevalence of thyroid disorders in the working population of Germany: ultrasonography screening in 96,278 unselected employees. Thyroid 200414 926-932. (doi:10.1089/thy.2004.14.926)

10 Jin J \& McHenry CR. Thyroid incidentaloma. Best Practice \& Research. Clinical Endocrinology \& Metabolism 201226 83-96. (doi:10.1016/ j.beem.2011.06.004)

11 Steele SR, Martin MJ, Mullenix PS, Azarow KS \& Andersen CA. The significance of incidental thyroid abnormalities identified during carotid duplex ultrasonography. Archives of Surgery 2005140 981-985. (doi:10.1001/archsurg.140.10.981)

12 Pieterman CR, Conemans EB, Dreijerink KM, de Laat JM, Timmers HT, Vriens MR \& Valk GD. Thoracic and duodenopancreatic neuroendocrine tumors in multiple endocrine neoplasia type 1: natural history and function of menin in tumorigenesis. Endocrine-Related Cancer 2014 21 R121-R142. (doi:10.1530/ERC-13-0482)

13 Dong Q, Debelenko LV, Chandrasekharappa SC, Emmert-Buck MR, Zhuang Z, Guru SC, Manickam P, Skarulis M, Lubensky IA, Liotta LA et al. Loss of heterozygosity at 11q13: analysis of pituitary tumors, lung carcinoids, lipomas, and other uncommon tumors in subjects with familial multiple endocrine neoplasia type 1. Journal of Clinical Endocrinology and Metabolism 199782 1416-1420. (doi:10.1210/jcem.82.5.3944)

14 Kim HJ, Park JS, Kim CS, Kang ES, Cha BS, Lim SK, Kim KR, Lee HC \& Ahn CW. A case of multiple endocrine neoplasia type 1 combined with papillary thyroid carcinoma. Yonsei Medical Journal 200849 503-506. (doi:10.3349/ymj.2008.49.3.503)

15 Desai D, McPherson LA, Higgins JP \& Weigel RJ. Genetic analysis of a papillary thyroid carcinoma in a patient with MEN1. Annals of Surgical Oncology 20018 342-346. (doi:10.1007/s10434-001-0342-8)

16 Vortmeyer AO, Lubensky IA, Skarulis M, Li G, Moon YW, Park WS, Weil R, Barlow C, Spiegel AM, Marx SJ et al. Multiple endocrine neoplasia type 1: atypical presentation, clinical course, and genetic analysis of multiple tumors. Modern pathology 199912 919-924. PMID: 10496602.
Received 20 October 2014

Revised version received 18 December 2014

Accepted 7 January 2015 